\title{
THE IMPACT OF ANTERIOR CRUCIATE LIGAMENT DEFICIENCY AND RECONSTRUCTION ON BILATERAL THIGH MUSCLE ACTIVITY DURING STAIR DESCENT
}

\begin{abstract}
Aim. To assess electromyographic (EMG) gait pattern changes during stair descent in subjects with chronic anterior cruciate ligament ( $A C L$ ) deficiency, and in subjects after $A C L$ reconstruction.

Methods. Thirteen ACL deficient subjects (ACLdef), 8 ACL reconstructed subjects (ACLrec), and 10 controls (CON) participated in the study. All subjects were assessed for functional and lean thigh volume (LTV) differences in the injured and uninjured limb. All subjects then stepped off a bench with EMG electrodes on the vastus medialis, vastus lateralis and hamstrings muscles of both limbs.

Results. Step down activity caused similar EMG responses in the injured and supporting limb in the ACLdef group compared to that in the control group. In contrast, in the ACLrec group, the onset of EMG activity occurred earlier during the step down activity in the vastus medialis and

ST CLAIR GIBSON A, MBCHB, PhD'; LAMBERT MI, PhD', VAUGHAN CL, PhD', LOWERY M, BSc(ENG) ${ }^{3}$, O'MALLEY MJ, PhD', NOAKES TD, MBCHB, MD'

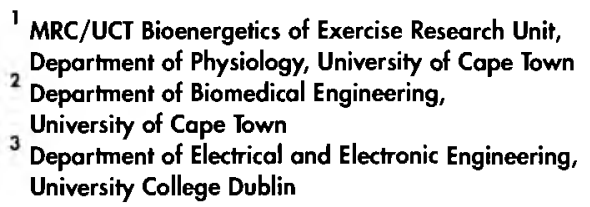
vastus lateralis of the supporting limb. There was significantly greater EMG activity in the vastus lateralis and hamstring muscles of the supporting limb $(p<0.05)$ in the ACLrec group compared to ACLdef and control groups. The changes in EMG activity did not appear to be related to differences in LTV, since greater LTV deficits were present in the ACLdef compared to the ACLrec group $(p<0.01)$.

Conclusions. In ACL reconstructed subjects, changes in muscle recruitment patterns in the supporting limb during step down activity have been identified. This altered activity pattern was not present in the supporting limb of ACL deficient subjects
\end{abstract}

KEY WORDS: GAIT PATTERN ACTIVITY, STEP DOWN, EMG

\section{INTRODUCION}

The control of electromyographic (EMG) gait patterns in normal individuals involved in walking activities and functional daily activities has been extensively examined (Ciccotti et al, 1994; Olree and Vaughan, 1995). The contributions of quadriceps and hamstrings muscles respectively in controlling movement around the knee joint is also well documented. The hamstring muscles act as a secondary stabilizer of the knee joint in the normal individual,

\section{CORRESPONDENCE TO:}

Dr A St Clair Gibson, MRC/UCT

Bioenergetics of

Exercise Research Unit

Sport Science Institute of South Africa

PO Box 115, Newlands, 7725

South Africa

Tel: 27-21-6867330

Fax: 27-21-6867530

Email: agibson@sports.uct.ac.za preventing anterior tibial translation during leg extension (Aune et al, 1995). The quadriceps musculature either increases or decreases anterior tibial translation forces depending on the angle of knee flexion (Andriacchi et al, 1992).

There are alterations in the normal muscle activity pattern after ACL injury (Solomonow et al, 1987). With ACL deficiency, the hamstrings muscles show increased activity during extension while the quadriceps muscle activity is inhibited during ballistic movements (Liu and Maitland 2000; St Clair Gibson et al, 2000 (a); St Clair Gibson et al, 2000 (b)). These changes may be related to increased anterior tibial translation, which occurs in ACL deficiency (Solomonow et al, 1987). Most (Gauffin and Tropp, 1985), but not all research (Pope et al, 1990) support these findings.

It has been suggested that there are centrally-mediated changes in gait patterns which may occur in both limbs (Hurwitz et al, 1997; Berchuk et al, 1990) as a "quadriceps avoidance behavior" to reduce stress on the ACL deficient knee. Others suggest that this protective behavior may be a learned response activated several years after ACL injury (DeVita et al, 1997). It has been shown that ACL reconstruction changes muscle activity towards a more normal gait pattern in the damaged limb (Ciccotti et al, 1994), although studies have shown either no improvement in quadriceps function (St Clair Gibson et al, 2000 (c)) or changes in hamstring activity in the damaged limb after ACL reconstruction (DeVita et al, 1997), depending on the testing protocol used.

The aim of this study, therefore, was to assess whether stair descent, which predisposes the descending limbs to anterior tibial translation, causes muscle pattern changes in both injured and uninjured limbs in subjects with ACL deficiency and in subjects who had undergone surgical ACL reconstruction. 


\section{METHODS}

\section{Subjects}

Thirteen subjects with ACL deficient knees, 8 subjects with ACL reconstructions and 10 control subjects participated in the trial. The method of recruitment for the ACL deficient (ACLdef) and ACL reconstructed (ACLrec) subjects was by advertisement in the local press, and by referral from orthopaedic surgeons. Inclusion criteria for the ACLdef subjects were: (i) the subjects had to have sustained an ACL rupture at least one or more years previously which had been diagnosed by an orthopaedic surgeon and which had been managed conservatively with no attempt at repair of the damaged ligament; (ii) subjects' contralateral knee joint had to be normal with no prior injury; and (iii) subjects had to be disease free. The only different inclusion criterion for the ACLrec group was that the subjects' ACL had to have been surgically reconstructed one year or more previously. The inclusion criteria for the control subjects (CON) was that they had no history of knee injuries or lower limb abnormalities in either limb. In all groups, because the subjects' normal limb served as an internal control, age and gender were not regarded as exclusionary criteria. The study was approved by the Ethics Committee of the University of Cape Town and all subjects signed an informed consent prior to the start of the trial.

\section{Magnetic resonance imaging (MRI) scan}

Each subject underwent an MRI scan (Esaote Biomedica Artoscan, Genoa, Italy) of the damaged knee to verify ACL deficiency and ACL reconstruction.

\section{Injury Score}

A detailed history was recorded from each subject, describing the episode which led to the ACL rupture and the post-injury symptomatology, using the Cincinnati functional rating scale (Noyes et al, 1984).

\section{Anthropometry}

Each subject's height and mass were recorded, and their body fat was assessed using the sum of the skinfold measurements of the right triceps, biceps, subscapular and supra-iliac skinfold sites (Durnin and Womersley, 1974). In addition, the anterior mid-thigh skinfold measurement, the sub-gluteal, mid-thigh and above-knee circumferences were recorded in both limbs to calculate the lean thigh volume (LTV) of the right and left legs. This technique for estimating LTV, which was adapted from Katch and Katch (1974), assumes the upper lower limb to have the shape of a truncated cone. The technique has been validated against LTV assessed by MRI (Knapik et al, 1996).

\section{Step down activity}

The subjects in all groups were instructed to step down off a single wooden step $25 \mathrm{~cm}$ in height. The same wooden step was used for all subjects in the trial. The subjects were familiarised with the step activity before beginning the trial, and each subject performed three warm up step downs leading with both left and right feet.

Once the subjects were familiar with the testing protocol, they were instructed to remain standing motionless on the step apparatus. On being given the command to begin, the subjects initiated movement with their ACL deficient/ reconstructed limb and in a regular process descended to the ground leading with their ACL deficient/reconstructed limb. The test was concluded when the supporting foot had reached the ground and the subjects were again stationary. In the results and discussion section, the descending limb is defined as the foot initiating step down activity and the supporting limb defined as the limb remaining on the step until toe strike and stance phase of the descending limb occurs.

\section{Electromyographic (EMG) activity}

Prior to the step down activity, EMG electrodes were attached to the subjects' lower limbs. The vastus medialis (VM), vastus lateralis (VL) and hamstrings (HAM) muscles of both legs were the muscle groups used for EMG assessment.

The electrode was positioned over the main belly of VM $8 \mathrm{~cm}$ proximal to the superior border of the patella in a line measured from the middle of the superior border of the patella to the anterior superior iliac spine, and $5 \mathrm{~cm}$ medial to this line in a perpendicular direction.

The position of the electrode on the VL was $3 \mathrm{~cm}$ anterior to the midpoint of the line between the lateral femoral epicondyle and the greater trochanter of the femur. The position of the electrode on the HAM muscles was at the midpoint of the line between the lateral femoral epicondyle and the greater trochanter of the femur in the axis of the line between the ischial tuberosity and the midpoint of the posterior aspect of the knee joint.

The skin overlying the muscles was carefully prepared. Hair was shaved off, the outer layer of epidermal cells abraded, and the remaining oil and dirt were removed with an alcohol pad. Triode electrodes (Thought Technology TriodeTM MIEP01-00) were placed on the muscle positions described previously and linked via fibre-optic cable to the Flexcomp/DSP EMG apparatus (Thought Technology, Montreal, Canada) and host computer. Each step down activity was sampled at $1984 \mathrm{~Hz}$ for the duration of the test, thus yielding raw EMG signals. A toggle switch was activated at the beginning of each test to mark the startpoint of the test procedure.

Movement artefact was removed from the raw EMG signal using a highpass second order Butterworth filter with a cut off frequency of $15 \mathrm{~Hz}$. The signal was full-wave rectified and then smoothed with a low-pass second-order Butterworth filter with a cut-off frequency of $5 \mathrm{~Hz}$ (Winter, 1984). Normalisation of the data was based on the toggle-switch data using the descending limbs contact with the floor as the reference point, and by dividing each data point by the maximal value after full wave rectification and smoothing elicited during the step down procedure (Winter, 1994). The damaged limb in the ACL subjects and the left limb of the control subjects were used as the leading limb.

\section{Statistics}

Total area under the curve for EMG activity data vs. time was analyzed prior to normalization of the data using Graphpad Prism software (GraphPad Software, Inc., San Diego). All data are 
FIGURE 1. The normalised, averaged EMG activity in the vastus medialis, vastus lateralis and hamstrings of both the descending (1) and supporting (2) limbs in the control subjects during step down activity. The dashed line marks the point at which the descending foot makes contact with the ground.

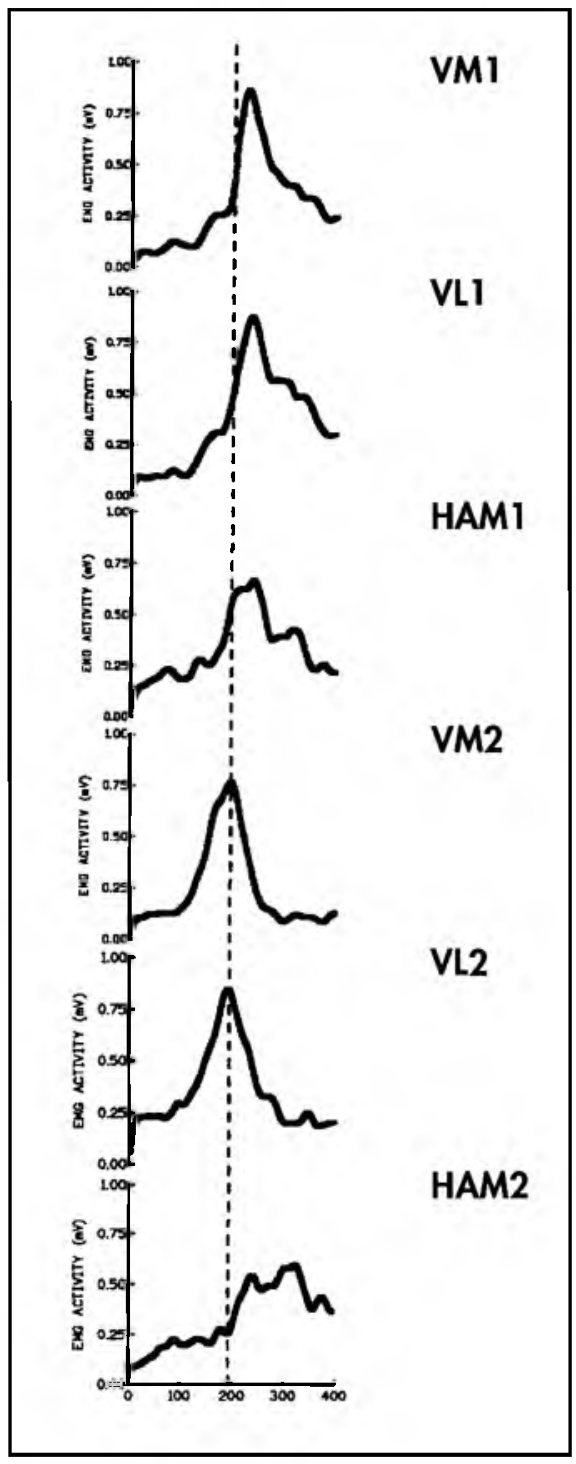

expressed as the difference between left and right limbs in the normal subjects and injured and uninjured limbs in the ACL subjects. A Kruskal Wallis test was used to assess differences between groups for the non-parametric data. An analysis of variance and Scheffe's posthoc test was used to compare differences between limbs of each group for anthropometrical and EMG data. Statistical significance was accepted when $\mathrm{p}<0.05$.

\section{RESULTS}

Table 1 shows the descriptive data of the subjects in the trial. There were no

TABLE 1. Descriptive data of subjects in the control (CON); $A C L$ deficient (ACLdef) and $\mathrm{ACL}$ reconstructed (ACLrec) groups.

\begin{tabular}{|l|c|c|c|c|}
\hline & Age & Height $(\mathrm{cm})$ & Weight $(\mathrm{kg})$ & Body Fat (\%) \\
\hline CON & $24.2 \pm 3.2^{* *}$ & $173.9 \pm 8.3$ & $68.6 \pm 13.0$ & $19.3 \pm 6.4$ \\
\hline ACLdef & $37.8 \pm 11.5$ & $172.5 \pm 8.1$ & $74.9 \pm 14.3$ & $22.3 \pm 7.2$ \\
\hline ACLrec & $35.9 \pm 6.1$ & $174.1 \pm 6.8$ & $79.1 \pm 13.0$ & $24.1 \pm 5.2$ \\
\hline
\end{tabular}

All data are mean $\pm \mathrm{SD}$

$\begin{array}{lll}* *-p<0.01 & \text { Age } & \text { CON vs. ACLrec } \\ & \text { Age } & \text { CON vs. ACLrec }\end{array}$

significant differences between height, weight, and percentage body fat between the three groups. The CON group was significantly younger than the ACLdef and the ACLrec groups ( $p<0.01)$. The subjects in the ACLrec group had undergone the following surgical procedures: five bone-patellar tendon-bone reconstructions, 2 semitendinosus reconstructions and 1 primary repair reconstruction.

Both the ACLdef and ACLrec groups had significantly lower scores than the CON group for the Cincinnati functional rating scale $(62.0 \pm 10.0$ vs $83.6 \pm 7.2$ vs $100.0 \pm 0.0$; ACLdef vs ACLrec vs CON; $\mathrm{p}<0.01$ ). The ACLrec group had significantly higher total scores than the ACLdef group ( $p<0.01$ ).

The difference in LTV between limbs was significantly greater in the ACLdef group than the CON group $(3 \pm 84$ vs $416 \pm 276 \mathrm{cc}$; CON vs ACLdef; $\mathrm{p}<0.01)$. Although the difference in LTV between limbs was greater in the ACL rec group than the CON group, the differences were not significant $(3 \pm 84$ vs $238 \pm 224$ cc; CON vs ACLdef)

There were no significant differences between ACLdef, ACLrec and CON groups for area under the curve EMG values in VM, VL and HAM muscles of the descending injured limb during step down activity.

There were no significant differences between ACLdef, ACLrec and CON groups for area under the curve EMG values in the VM muscle of the supporting limb during step down activity. The area under the curve EMG values were significantly higher in the ACLrec than both ACLdef and CON groups for both the VL $(17223 \pm 6004$ vs $9632 \pm 7302$ vs $7824 \pm 2281 \mathrm{mV}$; ACLrec vs ACLdef vs CON; p < 0.05) and HAM $(13740 \pm 4730$ vs $7436 \pm 3737$ vs 7264
$\mathrm{mV}$; ACLdef vs ACLrec vs CON; $\mathrm{p}<0.05$ ) muscles in the supporting limb.

The EMG gait pattern of control subjects during step down activity for the VM, VL and HAM muscles of both the descending and supporting limbs are depicted in figure 1 . The time point of the descending foot making contact with the floor and subsequently bearing weight is delineated by the dashed line. The maximal EMG activity in the descending limbs occurred immediately after toefloor strike for both VM and VL. The activity in HAM in the descending limb also reached maximal values soon after toe-strike, but showed more of a plateau than peak shape, compared with the VM and VL.

In the control subjects supporting limb, the timing of maximal EMG was earlier than that in the descending limb, and occurred at almost exactly the same time as toe strike of the descending limb in VM and VL. The shape was similarly a sharp peak with activity decreasing rapidly after toe strike. In the hamstring musculature the activity increased gradually then plateaued and was maintained after toe strike (Figure 1).

The activity patterns in the VM, VL and HAM muscles of the descending injured limb of the ACLdef and ACLrec were essentially similar to the CON group and are not illustrated.

Figure 2 depicts the EMG activity pattern in VM of the supporting uninjured limb. The activity patterns of the CON and ACLdef were similar. In the ACLrec group, the peak EMG activity in the VM occurred earlier, prior to the descending limbs contact with the floor, than in both the CON and ACLdef groups. Similar differences between ACLrec and ACL def and CON groups EMG activity patterns to those in the 
FIGURE 2. The normalised, averaged EMG activity in the vastus medialis of the supporting limb of the ACLdef, ACLrec andCON groups during step down activity. The dashed line marks the point at which the descending foot makes contact with the ground.

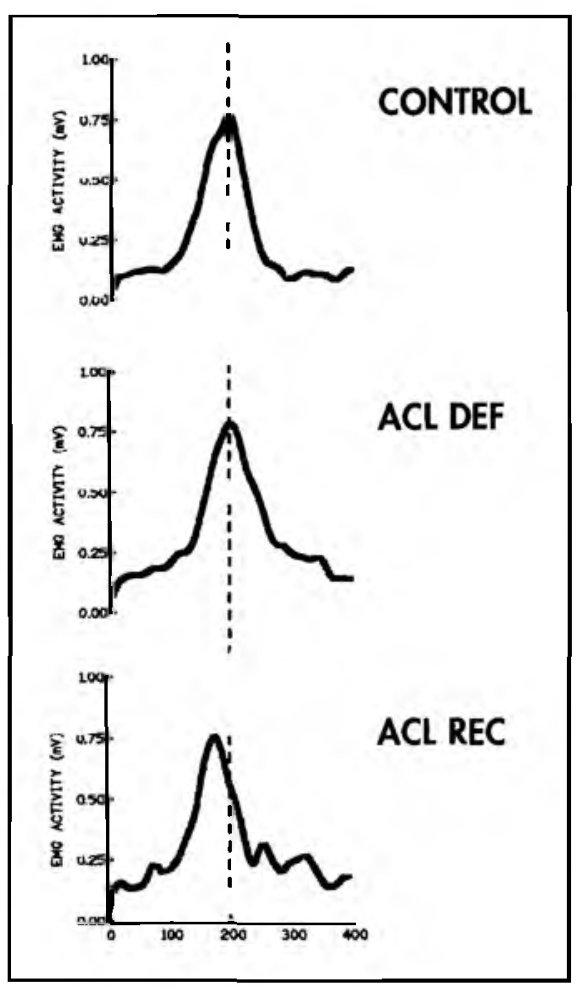

VM were found in the VL of the supporting limb (Figure 3). The EMG activity patterns in the HAM muscles of the supporting limb was similar in all groups.

\section{DISCUSSION}

The first important finding was that there were differences in the EMG activity patterns of the supporting limb in the ACL reconstructed group, with an increased amount of EMG activity in the vastus lateralis and hamstring muscles and earlier firing in the vastus medialis and vastus lateralis muscles. A possible explanation for these differences in EMG activity was that these changes were an attempt by the supporting limb to reduce the force placed on the damaged limb during the step down activity. This would protect the ACL reconstruction from increased anterior tibial translation in the ACL reconstructed knee after toe strike. Similar functional and EMG activity pattern changes following $\mathrm{ACL}$ reconstruction have previously been described in the litera-
FIGURE 3. The normalised, averaged EMG activity in the vastus lateralis of the supporting uninjured limb of the ACLdef, ACLrec and CON groups during step down activity. The dashed line marks the point at which the descending foot makes contact with the ground.

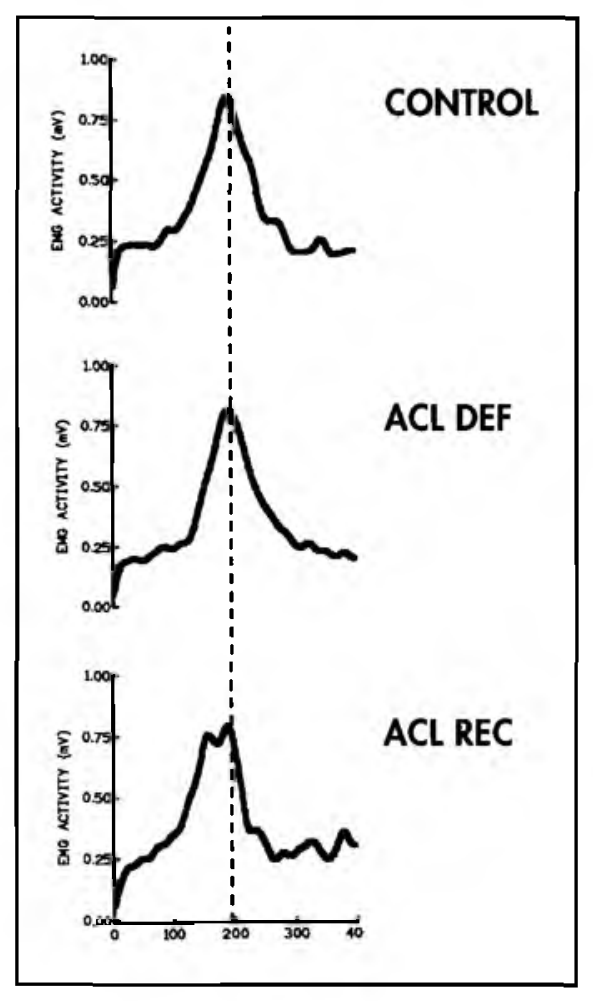

ture (DeVita et al, 1997; Pfeifer, 1997). Several researchers have hypothesized that graft harvesting intra-operatively causes changes in EMG patterns in both limbs (Pfeifer, 1997; Kowalk et al, 1997). Another explanation for this EMG activity pattern change could be that there is a learned protective response which occurred in the short-term rehabilitative phase after ACL reconstruction was performed. It has even been suggested that the altered gait patterns may be a learned response to long term use of a functional knee brace after ACL reconstruction (DeVita et al, 1997).

The finding that this protective avoidance pattern occurred only in the ACL reconstructed group and not in the ACL deficient group is significant. The changes which were described previously as an inhibitory quadriceps response, which occurs during isokinetic movement and is present in ACL deficient groups damaged limb as opposed to supporting limb (Solomonow et al, 1987; St Clair Gibson et al, 2000 (c)), is likely to have a different underlying mecha- nism. The mechanism in this study can be interpreted as a protective excitatory response in the supporting uninjured limb occurring after ACL reconstruction, with the mechanism identified by Solomonow et al (1987) is more likely to be a reflex inhibitory response of the quadriceps muscles in the injured limb of chronic ACL deficient individuals.

In contrast to the findings of this study, Bulgheroni et al (1997) found that during treadmill walking ACL reconstructed subjects had normal muscle activity patterns, while ACL deficient subjects had altered muscle activity patterns. Similarly, a "quadriceps avoidance gait" (Berchuk et al, 1990) and changes in hamstring firing patterns (Ciccotti et al, 1994) have been described in ACL deficient subjects during flat treadmill walking. This finding was not present in the ACL deficient subjects used in our trial. These contradictory findings may be due to differences in testing protocols - walking on a flat straight surface in the study by Bulgheroni et al (1997) rather than the step down activity used in this study.

Step down activity caused no significant changes in EMG activity in the vastus medialis, vastus lateralis or hamstring muscles of the descending limb of the ACL deficient group. This finding contradicts the EMG changes described in previous studies of ACL deficient subjects (Solomonow et al, 1987). The discrepancies in these findings may be due to the fact that loading of the ACL is angle-specific (Shelburne and Pandy, 1997), and the knee joint angles traversed in step down activity did not stress the knee joint sufficiently to evoke the ACL - quadriceps response described by other investigators (Gauffin and Tropp, 1985). Indeed, it has been demonstrated that beyond a flexion angle of $70 \mathrm{o}$, quadriceps contraction paradoxically reduces strain on the ACLduring step activities (Hurwitz et al, 1997).

Although there was a significantly decreased LTV in the ACL deficient limb, there were no significant differences in LTV in the supporting limb in either the ACL deficient or ACL reconstructed groups, indicating that the EMG pattern changes in the supporting limb were not caused by changes in muscle 
size. Although LTV decreases between limbs were significantly greater in the ACL deficient group than in the ACL reconstructed group, there were no changes in either the quantity or pattern of EMG activity. As stated previously, the step down activity may not have applied sufficient force to the damaged limb to produce the changes in EMG activity described by Solomonow et al (1987) in the ACL deficient limb.

It must be noted that the ACL reconstructed group was a fairly heterogeneous, small sample size. Kinematic analysis was not performed of the subjects limbs during step down activity, thus the speed at which the step down was performed was not assessed. The ACL reconstructed subjects may have stepped down more slowly then the ACL deficient and control groups, which may alter the pattern and relative dynamic activation of the different muscle participating in the movement. The changes may therefore have been caused by a change of speed of stepping rather than an actual re-organization of the muscle activity patterns in the ACL reconstructed group. Further studies therefore should examine a larger sample size and control for speed of stepping using kinematic analysis.

It must be noted also that the control group was significantly younger than either the ACL reconstructed or deficient groups. However, as described earlier, as the subject's normal limb served as internal controls, and because ACL deficient individuals has similar results to the control group, it is unlikely that the differences in the ACL reconstructed group were caused by age associated changes in muscle activity.

From a clinical perspective, it would appear that the changes in the ACL reconstructed group are related to processes occurring either during the operative reconstruction or post-operative rehabilitation which may have been different compared to that in the ACL deficient group. Although the step down activity patterns in the ACL reconstructed group are different to those in the control group, it would appear that they serve a protective function teleologically. As previous studies have shown that ACL reconstructed subjects suffer fewer episodes of giving way and less functional disability (St Clair Gibson et al 2000 (c)), the changes in activity in the supporting limb may be beneficial. Therefore, physiotherapy protocols aimed at normalizing these altered muscle patterns may be counter-intuitive. However, it is not clear from this study whether these altered firing patterns would predispose the supporting limb to future injuries. Further research is required to assess the injuries to the supporting limb after ACL reconstruction.

In conclusion, in ACL reconstructed subjects, the uninvolved supporting limb appears to have altered muscle activity patterns during step down activity. In contrast step down activity does not alter muscle activity patterns in ACL deficient subjects. Further research is needed to assess the reason for these different findings in ACL reconstructed and ACL deficient individuals.

\section{ACKOWLEDGEMENTS}

The Medical Research Council (MRC) of South Africa and the Duncan Baxter Research Fund of the University of Cape Town supported this study.

\section{REFERENCES}

Andriacchi TP, Birac D. 1992 Functional testing in the anterior cruciate ligament deficient knee. Clinical Orthopaedics 2:40-47

Aune AK, Ekeland A, Nordsletten L. 1995 Effect of quadriceps or hamstrings contraction on the anterior shear force to anterior cruciate ligament. An in vivo study. Acta Orthopaedica Scandinavica 66:261-265

Berchuk M, Andriacchi TP, Bach BR, Reider BR. 1990 Gait adaptations by patients who have a deficient ACL. Journal of Bone and Joint Surgery 72:871

Bulgheroni $\mathrm{P}$, Bulgheroni MV, Andrini L, Suffanti P, Giughello A. 1997 Gait patterns after anterior cruciate ligament reconstruction. Knee Surgery Sports Traumatology and Arthroscopy 5:14-21

Ciccotti MG, Kerland RK, Perry J, Pink M. 1994 An electromyographic analysis of the knee during functional activities. II. The anterior cruciate ligament-deficient and reconstructed profiles. American Journal of Sports Medicine 22:65I-658
DeVita P, Hortobagyi T, Barrier J, Torry M, Glover KL, Speroni DL, Money J, Mahar MT. 1997 Gail adaptations before and after anterior cruciate ligament reconstruction surgery. Medicine and Science in Sports and Exercise 29:853-859

Durnin JVGA, Womersley J. 1974 Body fat assessed from total body density and it's estimation from skinfold thickness. Measurements on 481 men and women aged from 16 to 72 . British Journal of Nutrition 32:77-79

Gauffin H, Tropp H. 1985 Altered movement and muscular-activation patterns during the one-legged jump in patients with an old anterior cruciate ligament rupture. Journal of Bone and Joint Surgery 67:1034-1043

Hurwitz DE, Andriacchi TP, Bush-Joseph CA, Bach BR. 1997 Functional adaptations on patients with ACL deficient knees. Exercise and Sports Science Reviews 25:1-20

Katch VL, Katch FI. 1974 A simple anthropometric method for calculating segmental leg limb volume. Research Quarterly 45:211-214

Knapik JJ, Staab JS, Harman EA 1996. Validity of an anthropometric estimate of thigh muscle cross-sectional area. Medicine and Science in Sports and Exercise 28:1523-1530

Kowalk DL, Duncan JA, McCue FC, Vaughan CL. 1997 Anterior cruciate ligament reconstruction and joint dynamics during stair climbing. Medicine and Science in Sports and Exercise 29:1406-1413

Liu W, Maitland ME. 2000 The effect of hamstring muscle compensation for anterior laxity in the ACL-deficient knee during gait. Journal of Biomechanics 33:871-879

Noyes FR, McGinnis GH, Mooar LA. 1984 Functional disability on the anterior cruciate insufficient syndrome. Review of knee rating systems and projected risk factors in determining treatment. Sports Medicine 1:278-302

Olree KS, Vaughan CL. 1995 Fundamental patterns of bilateral muscle activity in human locomotion. Biological Cybernetics 73:409-414

Pfeifer K. 1997 Functional dynamic tests in knee rehabilitation. 2nd Annual Congress European College of Sport Science Abstracts 264-265

Pope DF, Cole KJ, Brand RA. 1990 Physiologic loading of the anterior cruciate ligament does not activate quadriceps or hamstrings in the anaesthetized cal. American Journal of Sports Medicine 18:595-599 
Shelburne KB, Pandy MG. 1997 A musculoskeletal model of the knee for evaluating ligament forces during isometric contractions. Journal of Biomechanics 30:163-176

Solomonow M, Baratta R, Zhou BH, Shoji H, Bose W, Beck C, D'Ambrosia R. 1987 The synergistic action of the anterior cruciate ligament and thigh muscles in maintaining joint stability. American Journal of Sports Medicine 15:207-213

St Clair Gibson A, Lambert MI, Myburgh KH, Noakes TD. 2000 Quadriceps and hamstring function in complete and partial anterior cruciate ligament deficiency. South African Journal of Sports Medicine 2: 21-26 (a)

St Clair Gibson A, Lambert MI, Durandt JJ, Scales N, Noakes TD. 2000 Quadriceps and hamstrings peak torque ratio changes in persons with chronic anterior cruciate ligament deficiency. Journal of Orthopaedic and Sports Physical Therapy 30:418-427 (b)

St Clair Gibson A, Lambert MI, Myburgh KH, Walters J, Vaughan CL, O'Malley MJ, Noakes TD. 2000 The relationship between functional capacity, muscle size and strength in chronic ACL deficient and ACL reconstructed individuals. South African Journal of Physiotherapy 56:12-18 (c)

Winter DA. 1984 Pathologic gait diagnosis with computer-averaged electromyographic profiles. Archives of Physical Medicine and Rehabilitation 65:393-398

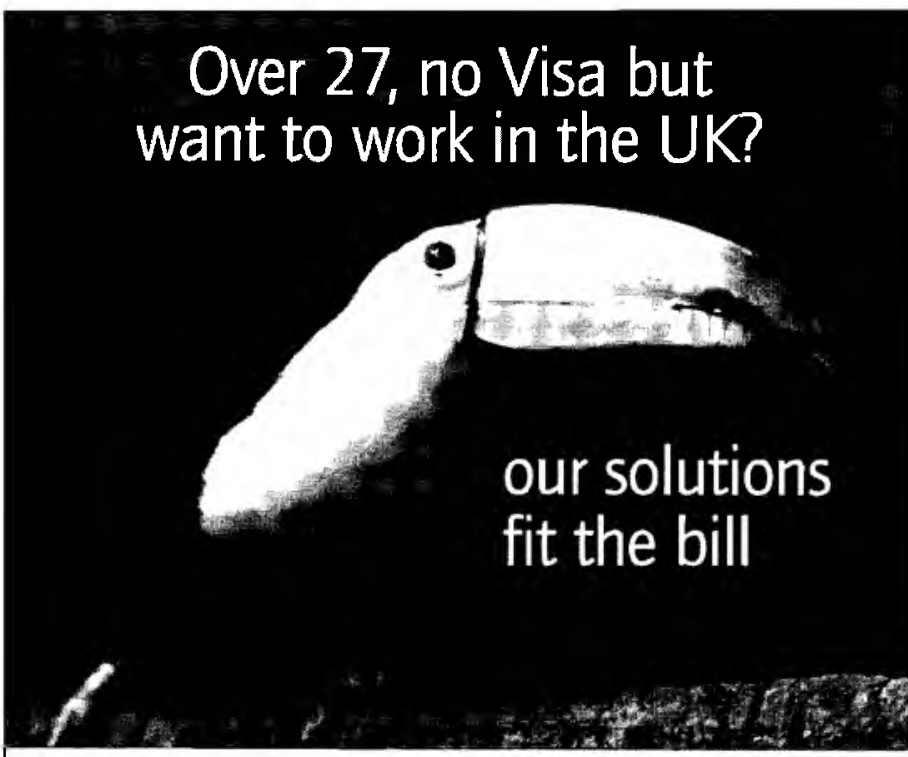

Physiotherapists

- Work Permits / Sponsorship arranged

- Assistance with UK State Registration

- Nationwide selection of vacancies

- All grades especially Senior and Management

Contact Ruth or Farhana Toll-Free on

0800-99-3055

Fax: 09442082361709

E-mail: permanent@corinth.co.uk www.corinth.co.uk

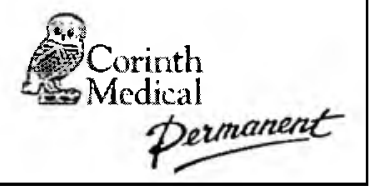

Physiotherapists wo... planning to...

We will help you make a positive choice of Physiotherapy locum or permanent positions. CMS offer a personal and professional service accompanied by a full and comprehensive range of benefits with particular emphasis on providing you with continual support whilst you are working in the UK.

contact Cecily Smyth BSc.0T

our S.A. Consultant in Jo'burg email: cecilysmyth@visto.com tel/fax: (II) 702 I375

(or visit our website) www.countrywidemedical.co.uk

\section{COUNTRYWIDE MEDICAL SELECTION}

Eastgate House, Eastgate Street, Winchester,

Hampshire SO23 8DZ England

Tel: $\quad$ Toll Free $0800999 / 48$

Fax:

$+441962865654$

Email: cms@cms-Itd.co.uk 